\title{
Changes in Behavior and Attentional Capacity after Adenotonsillectomy
}

\author{
BARBARA C. GALLAND, PATRICK J. DAWES, E. GAIL TRIPP, AND BARRY J. TAYLOR
}

\author{
Department of Women's \& Children's Health [B.C.G., B.J.T.], Department of Otorhinolaryngology, Head \& Neck Surgery [P.J.D.], \\ Department of Psychology [E.G.T.], University of Otago, Dunedin, New Zealand
}

\begin{abstract}
The objective of this study was to quantify behavioral and attention capacity changes in children aged $4-11$ y before and 3 mo after adenotonsillectomy (A/T). Overnight cardiorespiratory recordings were performed in 61 "behaviorally normal" children 1 wk before A/T. Tests of sustained attention using visual and auditory continuous performance tests (CPT) were completed by children 1 wk before and 3 mo after A/T. Behavioral Assessment Scales for Children (BASC) and a sleep questionnaire were completed by the parent/s at these same times. Results from overnight cardiorespiratory recordings showed that the children had mild sleeprelated breathing disorders (SRBD) preoperatively with a mean apnea/hypopnea index of 3.0/h and a movement awakening index of $2.5 / \mathrm{h}$. The majority had parent-perceived sleep and breathing difficulties that significantly improved post-A/T. BASC $T$ scores for externalizing and internalizing behaviors improved post-A/T, e.g., behavioral symptom index mean pre-A/T was 56.2 (95\% confidence interval, 52.8-59.6) compared with 50.9 (48.5-53.5) post-A/T. Some measures indicative of impulsivity and attentiveness obtained from the visual CPT before surgery, improved post-A/T, but no change was observed in any auditory CPT measures. Our data confirm improvements in subjective measures of sleep problems in children treated for SRBD and strengthen the notion of treating the disorder, not only related to the obvious clinical condition but also to the underlying sleep problems and adverse effects on daytime behavior and attention. (Pediatr Res 59: 711-716, 2006)
\end{abstract}

$\mathrm{C}$ hildren with adenotonsillar hypertrophy are at risk of suffering SRBD (1), with snoring, difficulty breathing, apnea, and persistent mouth breathing that ranges from primary snoring, through upper airways resistance syndrome, to OSA. The most common treatment for children with adenotonsillar hypertrophy or recurrent tonsillitis is $\mathrm{A} / \mathrm{T}$. This has been shown to result in clinical improvement in the majority of cases. In severe cases involving OSA, failure to treat can be associated with serious morbidity such as failure to thrive and systemic hypertension, and pulmonary hypertension with or without cor pulmonale can develop (1).

SRBD have been linked to daytime behavioral problems, predominantly those associated with hyperactivity, aggression, and inattention (2-4), and poorer performance on tests of memory and intelligence (5). At short-term follow-up after

Received June 2, 2005; accepted January 4, 2006.

Correspondence: Barbara Galland, Ph.D., Department of Women's \& Children's Health, University of Otago, P.O. Box 913, Dunedin, New Zealand; e-mail: barbara.galland@stonebow.otago.ac.nz

This study was supported by a University of Otago research grant.

DOI: 10.1203/01.pdr.0000214992.69847.6f treatment, significant improvements in children's vigilance and behavior in areas of aggression, inattention, impulsivity and hyperactivity $(6,7)$, and quality of life (8) have been reported. Also reported are short-term improvements in several cognitive factors such as perceptual closure, inference, organization, visual and auditory short-term memory, ability to solve problems and think analytically (9), and learning $1 \mathrm{y}$ after treatment for a SRBD in poor academic achievers (10).

The main physiologic characteristics during sleep for children with SRBD are intermittent hypoxemia associated with frequent apneas, hypopneas and sleep fragmentation due to frequent arousals, awakenings, and a reduction in REM sleep, all of which may impact adversely on neurobehavioral functioning (11). These adverse effects are well documented, although only a few small prospective studies have been carried out. The aim of this study was to quantify the behavioral and attention changes in children aged 4-11 y, before and 3 mo after surgery, and to examine any potential relationships between the severity of SRBD and changes in the main outcomes of behavior and attention.

\section{METHODS}

Subjects. The sample comprised 61 children aged 4-11 y. Subjects were recruited from a surgical waiting list for $\mathrm{A} / \mathrm{T}$ following referral by an otolaryngologist; $19(31 \%)$ because of obstructive airway symptoms relating to adenotonsillar hypertrophy and $42(59 \%)$ because of recurrent episodes of tonsillitis. Tonsillar size was graded according to the reduction in pharyngeal diameter at the fauces (12): Grade I, $0-25 \%$; II, 26-50\%; III, 51-75\% and; IV, 76-100\%. Exclusion criteria were gross sensory or motor problems, significant developmental delay, or previous diagnosis of attention deficit hyperactivity disorder (ADHD) or taking medication for ADHD. Recruitment success was 55\%. Deprivation index scores [New Zealand (NZ) Dep2001] were calculated for each child's domicile using GeoStan NZ (version 2.1). This uses data extracted from the 2001 census dividing NZ into 10ths of distributions in relation to several indicators of socioeconomic deprivation. The Otago Ethics Committee approved the study. Informed written consent was obtained from parent/s of all participants and with assent from the child.

Overnight cardiorespiratory recordings. One week before adenotonsillectomy (pre-A/T), each child underwent one night of cardiorespiratory recording. Thoracic and abdominal movements were recorded by inductive plethysmography (Respitrace model 150; Respitrace Co., NY ); $\mathrm{SaO}_{2}$ and pulse signal by pulse oximetry (Nellcor N-200, Nellcor, Pleasanton, CA); nasal airflow by thermistry (MLT415, ADInstruments Pty. Ltd., Castle Hill, Australia); breath sounds by microphone placed over the sternal notch; and ECG

Abbreviations: ACPT, auditory continuous performance test; AHI, apnea hypopnea index; A/T, adenotonsillectomy; BASC, Behavioral Assessment Scale for Children; OSA, obstructive sleep apnea; $\mathbf{S a O}_{2}$, hemoglobin oxygen saturation; SRBD, sleep-related breathing disorder; TRT, total recorded time 
was recorded using standard position electrodes. All signals were relayed through an analog-to-digital converter (PowerLab, ADInstruments Pty. Ltd.). Sleep, movement, and awakenings were recorded using time-lapse video recordings with an infrared light source. Because there were no EEG or electrooculogram recordings, behavioral movement awakenings were quantified using video recording observations together with increases in heart rate. A movement awakening index was expressed as the total number of movement awakenings per hour of TRT, i.e., time from behaviorally observed sleep onset to morning awakening. Sleep efficiency and snoring were calculated as the percentage of behaviorally observed sleep time. Respiratory events were classified and their number, frequency and duration recorded. Obstructive apnea was defined as the absence of airflow with continued chest wall and abdominal movement for at least two respiratory cycles $(13,14)$. Obstructive hypopnea was defined as a decrease in airflow to less than 50\% baseline amplitude for at least two respiratory cycles (15). An obstructive AHI was defined as the total number of obstructive apneas, hypopneas, and mixed apneas per hour of behaviorally observed sleep time. On the basis of "normative data," an obstructive AHI index $\geq 1.5$ was chosen as the cutoff for outside the range of normal (15).

Sleep and breathing questionnaire. As for all questionnaires, the parent (predominantly mother) completed this 28 -item questionnaire $1 \mathrm{wk}$ before surgery (pre-A/T) and 3 mo after (post-A/T). Questions were based on clinical experience and published signs and symptoms of children with SRBD with particular reference to a parent questionnaire designed by Brouillette et al. (16) for discriminating between children with and without proven SRBD from polysomnography and included questions about child and family history of breathing difficulties during sleep, sleep hygiene, snoring, enuresis, sweating during sleep, periodic limb movements, levels of household smoking, and daytime signs of sleepiness. Responses to the main questions were categorized as never/sometimes/often/always, with room for additional comments where appropriate.

$\boldsymbol{B A S C}$. The BASC Parent Rating Scales (17), a 131- to 138-item questionnaire that assesses a child's adaptive and problem behaviors in home and community settings, were completed pre- and post-A/T. Two forms were used, targeted at children aged 4-5 y and 6-11 y. These contain descriptors of behaviors that respondents rate on a 4-point scale ranging from "never" to "almost always." The scores yield a clinical profile and an adaptive profile (for 6-11 y olds). $T$-scores calculated represent a standard score normalized for the age of the child using a mean of 50 and a SD of 10. Test-retest reliability coefficients for the parent-completed rating scale over a $2-8 \mathrm{wk}$ interval range from 0.41 to 0.94 (17).

Tests of sustained attention. Visual and auditory continuous performance tests (CPT) taking 15 min each were used to measure sustained attention preand post-A/T, but were available to 6 - to 11 -y-old children only $(n=39)$. The Visual Conners' Continuous Performance Test (VCPT) II (18) is a computerized tool where stimuli (white letters) appear on a black screen for $250 \mathrm{~ms}$ at varying interstimulus intervals (ISI; 1, 2, and 4 s). Children respond by pressing the space bar immediately, except when the target letter $X$ appears. The test measures reaction time, inattention, impulsivity, and variability of reaction time. Test-retest reliabilities over 3 mo range from 0.55 to 0.84 for the most relevant outcomes (18).

The Auditory Continuous Performance Test (ACPT) (19) required children to listen to a string of 120 words repeated six times over, and indicate to the experimenter, whenever they heard the target word dog. Total error scores were calculated from the sum of omissions (inattention error) and commissions (impulsive error), and a vigilance decrement calculated [difference in percentage of responses to $d o g$ in the first presentation to the last (sixth)]. Test-retest reliability show moderate coefficient scores of 0.67 and 0.74 for children aged 7 and 9 y, respectively (19).

Statistical analyses. Statistical analyses were performed using STATA version 8.0 (StataCorp, College Station, TX). Nonparametric tests using Spearman's correlation coefficients were used to look for correlations between cardiorespiratory variables with continuous data. Standard logistic regression was used to investigate potential relationships between AHI $(<$ or $\geq 1.5)$, tonsillar size (grades 1 and II or III and IV), and other cardiorespiratoryderived variables. To investigate whether variables suggestive of a SRBD (pre-A/T) could predict behavioral scores, standard logistic regression was used for AHI $(<$ or $\geq 1.5)$ and ANOVA for $\mathrm{SaO}_{2}$ nadir, the movement awakening index, and percentage of recording snoring (transformed into tertiles). For sleep and breathing questionnaire-derived responses, $\chi^{2}$ analyses were performed (dichotomous outcomes), and pre-and post-treatment were compared using binomial statistics. Comparisons of pre- and post-treatment indices for behavior and attention, and deviations from the norm, were made with paired $t$ tests with $p$ values adjusted for unequal variances where appropriate. A value of $p<0.05$ (two-tailed test) was considered statistically significant.

\section{RESULTS}

Group characteristics. The sample of 61 children (35 boys/26 girls) ranged in age from 4 to $11 \mathrm{y}$ with a mean $\pm \mathrm{SD}$ of $7 \pm 2$ y. Most had a normal body mass index $(18.1 \pm 4.0$ $\mathrm{kg} / \mathrm{m}^{2}$ ), although $24 \%$ were overweight or obese for their age according to Cole et al. (20). Tonsillar size was graded I, II, III, and IV in $8,36,50$, and $6 \%$ of the sample, respectively. Smoking by at least one person was present in the households of $47 \%$. Children came from the full spectrum of domiciles, ranging from the $10 \%$ least deprived areas indicated by a NZDep2001 score of 1 , to the $10 \%$ most deprived areas scoring 10 ; mean $\pm \mathrm{SD}=4.8 \pm 2.9$. There was no evidence that the sample was drawn from a population with different frequencies from the overall population $\left(\chi^{2}=8.65, p=\right.$ 0.471).

Cardiorespiratory findings. Results are summarized in Table 1. Using an AHI index $\geq 1.5$ for being outside the range of "normal" (15), 43 children (70\%) reached this cutoff. Of the 19 children with obstructive airway symptoms from clinical history and examination alone, 14 (74\%) reached this cutoff. Ten children had an AHI $\geq 5,21 \%$ of those presenting with obstructive airway symptoms and $16 \%$ with just a history of recurrent tonsillitis. There was a tendency for tonsillar size to be related to $\mathrm{AHI} \geq 1.5$ [odds ratio (OR), 2.10 ; $95 \%$ confidence interval (CI), 0.82-5.36). Mean sleep efficiency was $87 \%$, with only two children scoring $<70 \%$. Eighty-seven percent $(n=53)$ exhibited $\mathrm{S} \mathrm{Sao}_{2}$ nadir between 95 and 90; $13 \%(n=8)$ reached $<90 \%$. $\mathrm{SaO}_{2}$ nadir was inversely correlated with AHI $(r=-0.46, p<0.001)$ and the percentage of recorded snoring $(r=-0.27, p=0.05)$, and AHI was positively correlated with the percentage of recorded snoring $(r=0.33, p=0.015)$.

Sleep and breathing items. The results are summarized in Table 2. Data were analyzed only when complete pre-post data were available, as sometimes parents chose not to answer or missed answering a particular question that precluded inclusion in the pre-post data analyses. Sleep and breathing disturbance item responses ranging from "never" to "always" were frequently reported pre-A/T. Sixty-four percent of the sample was reported to snore "frequently or constantly," but there was no significant relationship between this and the presence of household smoking. There was a slight trend for reported snoring levels by the parent and those measured

Table 1. Cardiorespiratory-derived variables recorded pre-A/T

\begin{tabular}{lc}
\hline & $\begin{array}{c}\text { Mean } \pm \mathrm{SD} \\
(n=61)\end{array}$ \\
\hline Observed sleep latency (min) & $35.1 \pm 30.7$ \\
$\mathrm{TRT} \dagger$ (h) & $9.4 \pm 1.2$ \\
Behaviorally observed sleep time (h) & $8.2 \pm 1.1$ \\
Awake (\% TRT) & $12.5 \pm 6.6$ \\
Sleep efficiency (\% TRT) & $87.5 \pm 6.6$ \\
Movement awakening index (per hour TRT) & $2.5 \pm 0.9$ \\
Snoring (\% of behaviorally observed sleep) & $17.5 \pm 14.4$ \\
$\mathrm{SaO}_{2}$ nadir (\%) & $91.7 \pm 4.3$ \\
$\mathrm{AHI}$ (per hour of behaviorally observed sleep time) & $3.0 \pm 2.6$ \\
\hline
\end{tabular}

$\dagger$ TRT covers time from behaviorally observed sleep onset to morning awakening. 
Table 2. Parental reports of sleeping items pre- and post-A/T

\begin{tabular}{|c|c|c|c|c|c|c|}
\hline \multirow[b]{2}{*}{ Variable } & \multicolumn{2}{|c|}{ Pre-A/T } & \multicolumn{3}{|c|}{ Direction of change Post-A/T $(n)$} & \multirow[b]{2}{*}{$p$ Value } \\
\hline & $\begin{array}{c}\text { Never/sometimes } \\
{[n(\%)]}\end{array}$ & $\begin{array}{c}\text { Frequently/constantly } \\
{[n(\%)]}\end{array}$ & Worse & Same & Improved & \\
\hline Difficulty breathing & $40(75.5)$ & $13(24.5)$ & 0 & 41 & 12 & $<0.0005$ \\
\hline Snoring & $21(35.6)$ & $38(64.4)$ & 0 & 25 & 34 & $<0.0001$ \\
\hline Sleep with mouth open & $16(27.1)$ & $43(72.9)$ & 1 & 27 & 31 & $<0.0001$ \\
\hline Snorting & $45(80.4)$ & $11(19.6)$ & 1 & 45 & 10 & 0.01 \\
\hline Hard to wake & $40(66.7)$ & $20(33.3)$ & 3 & 39 & 18 & 0.001 \\
\hline Daytime sleepiness & $48(81.4)$ & $11(18.6)$ & 0 & 50 & 9 & 0.004 \\
\hline Restless sleep & $34(59.6)$ & $23(40.4)$ & 2 & 33 & 22 & $<0.0001$ \\
\hline Restless legs & $53(91.4)$ & $5(8.6)$ & 3 & 51 & 4 & NS \\
\hline Enuresis & $52(89.7)$ & $6(10.3)$ & 1 & 54 & 3 & NS \\
\hline Drenching sweats & $24(54.5)$ & $20(45.5)$ & 3 & 29 & 12 & 0.035 \\
\hline Headaches & $53(91.4)$ & $5(8.6)$ & 1 & 52 & 5 & NS \\
\hline
\end{tabular}

during the overnight recording to be related (OR, 1.05; 95\% CI, 1.0-1.1). Post-A/T, there were significant improvements in all items including drenching sweats. Less than $10 \%$ of children were reported to have restless legs, enuresis, and headaches "frequently or constantly" before or after surgery.

Behavior. BASC scores are given in Table 3. Before surgery (pre-A/T), the following variable scores within the clinical profile were significantly higher than the $T$ score mean (50), but still within average range: hyperactivity, aggression, depression, somatization, attentional problems, and composite scores for externalizing and internalizing behaviors including the behavioral symptom index. Other variables within the clinical profile scores (i.e., conduct problems, anxiety, atypicality, and withdrawal) were not significantly different from the $T$ score mean. Post-A/T behavioral variable changes significantly improved for all except conduct problems, and $T$ scores were no longer different from the mean. Adaptive profile scores were never significantly different from the $T$ score mean, pre- or post-A/T. Linear regression analyses to look for any association of cardiorespiratory variables pre-A/T with concomitant behavioral scores revealed no significant correlation.

Tests of attention. Table 4 gives a summary of the $T$ scores for the 11 VCPT variables. Comparisons with the normal population mean showed that significantly higher scores for commissions (indicator for inattention and impulsivity) and detectability (indicator for inattention) were present pre-A/T but were within the average range of a normal nonclinical score. Significant improvements were found after surgery. Lower scores for hit reaction time (HRT) SE by ISI were detected pre-A/T, suggesting the group showed more consistent responses in their ability to change their reaction time in response to changing ISI. No other measures were significantly different from the population norm.

Table 5 gives ACPT summary scores for total errors by age group. Comparison with normative mean data showed all, excluding age 7 , were within the range of normal and showed no change in attention abilities post-A/T. The group of 7-y-

Table 3. BASC parental-rated $T$ scores pre- and post-A/T

\begin{tabular}{|c|c|c|c|c|c|}
\hline Profile & Variable & $\begin{array}{c}T \text { score } \$ \text { pre-A/T, } \\
\text { mean }(95 \% \mathrm{CI}) \\
n=61\end{array}$ & $\begin{array}{c}T \text { score } \S \text { post-A/T, } \\
\text { mean }(95 \% \mathrm{CI}) \\
n=61\end{array}$ & $\begin{array}{c}\Delta \text { Between means } \\
(95 \% \mathrm{CI})\end{array}$ & $p$ Value \\
\hline \multirow[t]{12}{*}{ Clinical } & Externalizing behaviors composite & $56.2(52.8-59.6)^{*}$ & $50.9(48.5-53.5)$ & $-5.2(-8.5$ to -1.9$)$ & 0.003 \\
\hline & Hyperactivity & $56.0(52.8-59.2)^{*}$ & $50.2(47.7-52.7)$ & $-5.8(-8.5$ to -3.1$)$ & 0.0001 \\
\hline & Aggression & $54.6(51.7-57.6)^{*}$ & $49.8(47.2-52.5)$ & $-4.8(-7.9$ to -1.7$)$ & 0.003 \\
\hline & Conduct problems $\dagger$ & $51.0(47.9-54.1)$ & $50.0(47.2-52.8)$ & $-0.6(-2.7$ to 1.4$)$ & NS \\
\hline & Internalizing behaviors composite & $57.6(54.6-60.6) * *$ & $50.1(47.2-53.0)$ & $-7.5(-9.7$ to -5.2$)$ & $<0.0001$ \\
\hline & Anxiety & $52.7(49.9-55.4)$ & $49.8(47.4-52.1)$ & $-2.9(-5.1$ to -0.7$)$ & 0.01 \\
\hline & Depression & $54.4(51.3-57.6)^{*}$ & $48.4(45.9-50.8)$ & $-6.1(-8.4$ to -3.8$)$ & $<0.0001$ \\
\hline & Somatization & $59.9(56.2-63.6)^{*}$ & $51.4(48.5-53.5)$ & $-8.5(-11.7$ to -5.3$)$ & $<0.0001$ \\
\hline & Atypicality & $53.4(49.9-56.8)$ & $49.5(47.0-52.0)$ & $-3.9(-6.7$ to -1.1$)$ & 0.008 \\
\hline & Withdrawal & $53.1(49.9-56.4)$ & $47.5(44.9-50.1)$ & $-5.7(-8.2$ to -3.2$)$ & $<0.0001$ \\
\hline & Attention problems & $56.1(53.4-58.7)^{*}$ & $52.7(50.2-55.2)$ & $-3.4(-5.8$ to -0.1$)$ & 0.006 \\
\hline & Behavioral symptom index composite & $56.3(53.1-59.5)^{*}$ & $50.2(47.5-53.0)$ & $-6.0(-8.9$ to -3.2$)$ & 0.001 \\
\hline \multirow[t]{4}{*}{ Adaptive $\dagger$} & Adaptive skills composite & $49.3(45.1-52.3)$ & $51.9(59.0-54.8)$ & $2.6(-0.4$ to 5.6$)$ & NS \\
\hline & Adaptability & $48.7(45.1-52.3)$ & $51.6(49.1-54.1)$ & $2.9(-0.32$ to 6.1$)$ & NS \\
\hline & Social skills & $49.2(46.4-51.9)$ & $51.8(49.1-54.4)$ & $2.6(0.8$ to 4.5$)$ & NS \\
\hline & Leadership & $49.0(44.8-53.2)$ & $51.4(47.5-55.2)$ & $1.5(-0.08$ to 3.1$)$ & NS \\
\hline
\end{tabular}

$* p<0.05 ; * * p<0.0001$ significantly different from $T$ score mean norm of 50 .

$\uparrow$ 6- to 11-y-olds only $(n=34)$.

$\S T$ score cutoffs: Clinical profile: $41-59=$ average, $60-69=$ at-risk. Adaptive profile: $41-59=$ average, $31-40=$ at-risk. 
Table 4. Visual Continuous Performance Test

\begin{tabular}{|c|c|c|c|c|}
\hline Response & $\begin{array}{c}T \text { score pre-A/T mean } \\
(95 \% \mathrm{CI})\end{array}$ & $\begin{array}{c}T \text { score post-A/T mean } \\
(95 \% \mathrm{CI})\end{array}$ & $\begin{array}{c}\Delta \text { Between means } \\
(95 \% \mathrm{CI})\end{array}$ & $p$ Value \\
\hline \multicolumn{5}{|l|}{ Inattention summary } \\
\hline 1. Hit reaction time (HRT) & $48.3(44.2-52.5)$ & $47.7(42.9-52.6)$ & $-0.6(-6.9$ to 5.6$)$ & NS \\
\hline 3. $\%$ Commissions & $56.9(53.7-60.2)^{* *}$ & $51.4(47.2-55.6)$ & $-5.5(-10.7$ to -0.32$)$ & 0.04 \\
\hline 4. HRT SE & $48.5(44.8-52.2)$ & $48.8(45.1-52.6)$ & $0.3(-4.9$ to 5.5$)$ & NS \\
\hline 5. Variability & $48.4(44.4-52.4)$ & $49.9(46.7-53.1)$ & $1.5(-3.5$ to 6.5$)$ & NS \\
\hline 8. HRT SE by ISI & $45.7(42.6-48.8)^{*}$ & $50.4(45.8-54.9)$ & $4.7(-0.7$ to 10.1$)$ & NS \\
\hline \multicolumn{5}{|l|}{ Impulsivity summary§ } \\
\hline 9. Perseverations & $51.3(46.8-55.7)$ & $52.9(47.1-58.7)$ & $1.6(-5.5$ to 8.7$)$ & NS \\
\hline \multicolumn{5}{|l|}{ Vigilance summary } \\
\hline 10. HRT by block & $49.9(46.7-53.1)$ & $49.5(46.9-52.0)$ & $-0.47(-4.5$ to 3.5$)$ & NS \\
\hline 11. HRT SE by block & $49.1(45.6-52.6)$ & $47.9(45.3-50.5)$ & $-1.2(-5.5$ to 3.1$)$ & NS \\
\hline
\end{tabular}

$* p<0.05 ; * * p<0.0001$ significantly different from $T$ score general population mean norm of 50 .

$\$$ The numbered responses measure 1) average speed of correct responses; 2) response not given; 3) response given after $X$ appears; 4) response speed consistency; 5) inconsistency; 6) discriminatory power to detect $X$ and non- $X$ stimuli; 7) ability to change reaction time to changing ISI; 8) consistency of 7; 9) any reaction time $<100 \mathrm{~ms} ; 10$ ) change in reaction time over test duration; 11) change in response consistency over test duration.

$\S$ Impulsivity summary includes data for commissions and HRT.

olds had better attention abilities than the norm both pre- and post-A/T. No significant change in vigilance pre- versus post$\mathrm{A} / \mathrm{T}$ was found. There were no significant correlations between cardiorespiratory variables with attention test scores pre-A/T.

Relationship between indicators for SRBD and behavior scores. Analyses to predict whether variables suggestive of a $\mathrm{SRBD}$ (pre-A/T) (i.e., AHI, $\mathrm{SaO}_{2}$ nadir, movement awakening index, and percentage snoring) could predict behavioral scores resulted in one significant association and one trend worth reporting. Thus, AHI was significantly positively associated with hyperactivity $[50.8 \pm 10.4(\mathrm{SD})$ and $58.3 \pm 12.5$ for AHI $<$ and $\geq 1.5$, respectively; $p=0.03$ ] and there was a trend for a positive association with the behavioral symptom index $(51.7 \pm 7.4$ and $57.3 \pm 12.7$, respectively; $p=0.08)$. No other significant associations were found.

\section{DISCUSSION}

This study of behaviorally normal children referred for $\mathrm{A} / \mathrm{T}$ showed that several parent-rated indices of child behavior significantly improved 3 mo after surgery. Objective testing of children's sustained attention also showed improvements in some areas indicative of inattentive and impulsive symptoms. A higher level of age-adjusted scores for many of the behavioral indices found before surgery concurs with other reports where behaviors such as hyperactivity and aggression have also been associated with SRBD $(21,22)$. Owens et al. (22) have suggested that these externalizing behaviors may be in part reflective of daytime somnolence, and thus of an underlying sleep disturbance. Our subjective measures of the severity of the nighttime disruption to sleep and breathing, including daytime somnolence, certainly revealed marked improvements after surgery. However, we found no association of factors that looked at somnolence in particular with any of the higher behavioral scores reported before surgery.

Before proceeding further, there are some methodological limitations to this study that warrant comment. There was no concurrent control group and thus we cannot exclude the possibility that some of the attentional test improvements observed postoperatively were a practiced effect despite valid test-retest reliability data $(18,19)$. The behavioral questionnaire changes were parent-perceived. The parents could never be blinded to the procedure so belief and expectation may

Table 5. ACPT: total error score for age

\begin{tabular}{|c|c|c|c|c|c|c|}
\hline \multirow[b]{2}{*}{ Age } & \multirow[b]{2}{*}{ No. } & \multicolumn{3}{|c|}{ Total error scores (no. omissions + commissions) } & \multirow[b]{2}{*}{$\begin{array}{c}\Delta \text { Between means } \\
(95 \% \mathrm{CI})\end{array}$} & \multirow[b]{2}{*}{$P$ Value } \\
\hline & & $\begin{array}{c}\text { Normal } \\
\text { population mean } \dagger\end{array}$ & $\begin{array}{l}\text { Pre-A/T mean } \\
\quad(95 \% \mathrm{CI})\end{array}$ & $\begin{array}{c}\text { Post-A/T mean } \\
(95 \% \mathrm{CI})\end{array}$ & & \\
\hline 6 & 8 & 20.4 & $22.1(12.8-31.4)$ & $24.6(11.8-37.4)$ & $2.5(-11.8$ to 16.8$)$ & NS \\
\hline 7 & 8 & 16.2 & $8.5(1.1-15.9) *$ & $9.2(2.5-15.9) *$ & $0.7(-8.2$ to 9.7$)$ & NS \\
\hline 8 & 8 & 12.8 & $13.6(0-28.3)$ & $9.2(1.3-17.1)$ & $-4.5(-18.9$ to 9.9$)$ & NS \\
\hline $9-11 \div$ & 12 & 6.7 & $6.2(1.8-10.5)$ & $5.1(1.7-8.5)$ & $-1.0(-1.3$ to 3.1$)$ & NS \\
\hline
\end{tabular}

$* p<0.05 ; * * p<0.0001$ significantly different from ACPT data from a study of 220 children with normal attention abilities (19).

Repeat data were not available for three children.

$\dagger$ ACPT data from a study of 220 children with normal attention abilities (19).

$\ddagger$ Data pooled for 9- to 11 -y-olds because the normal population mean shows little variation over this age range (19). 
have played some part in the perceived improvements. Despite these limitations, our findings are consistent with the large body of evidence in the literature reporting an adverse association between SRBD and several facets of daytime functioning (2-5) together with data showing improvements after $\mathrm{A} / \mathrm{T}$ $(6-10)$.

In contrast to the externalizing and internalizing behaviors, measures of the children's adaptive skills and the sub-scales of adaptability, social skills, and leadership were all judged to be close to the norm before surgery and remained so at follow-up. Adaptive skills may be considered learned behaviors that appeared to be unaffected by the SRBD. We did not test specifically for cognitive processing, but a study of children age 5-9 y with longer term follow-up (6-10 mo) showed significant improvements in neurocognitive function including academic performance after adenotonsillectomy (9). Children with SRBD in whom the condition is left untreated have been suggested to be at risk of underachieving academically $(9,10)$. Improvements in learning have been reported after treatment $(3,10)$. However, the report that children who snore loudly and frequently during childhood risk poorer academic performance in later years, even well after the snoring has resolved, suggests that treatment may only reverse a learning deficit in the short term (23).

Our study showed that the VCPT revealed some mild impulsive and inattentive symptoms in children before surgery that reversed to the general population mean norm after surgery. The markers for inattentiveness included the detectability measure. This is a measure of a child's discriminatory power, suggesting that minor perceptual difficulties were evident before surgery. We acknowledge that repeatability of the task may have influenced our results, but the time frame of 3 mo was well outside of the 1-14 d test-retest reliability studies that showed moderate correlations (19). Also, our findings concur with others in that several studies have shown that children with SRBD have poorer measures of attention span compared with a control group $(3,9,21)$. Furthermore, Friedman et al. (9) have shown that the poor attention span in OSA-treated children, followed-up 6-10 mo after surgery, reversed compared with that of their control group. Avior et al. (7), using a comparable visual CPT tool in a small sample of children with OSA have shown 2-mo postoperative improvements in measures of both inattention and impulsivity. The auditory test we used, however, did not expose any deficits in attention, impulsivity, or vigilance. An anomaly was seen in the group of 7-y-olds who performed better than the norm for their age group before and after surgery and the 10 -y-olds after surgery. This particular age group had a bias toward good performance that was unavoidable with the small sample numbers.

Funding restrictions limited our ability to perform overnight cardiorespiratory studies at follow-up, but the positive result from the parental questionnaires designed to measure the symptoms and severity of a number of sleep and breathing items suggest overall improvement of sleep and breathing disturbances after A/T. The findings support other studies also based on parental reports $(9,24,25)$, although we acknowledge that parental perception of their child's resolution of SRDB can sometimes be wrong (26). However, it is well documented that as many as $80 \%$ of children have polysomnographically determined improved breathing during sleep and resolution of SRBD after A/T $(25,26)$, and our results would suggest from subjective measures that up to $60 \%$ improved depending on the breathing item chosen. Others have also shown significant improvements of many polysomnographic-derived variables $(6,27,28)$. A recent study evaluating polysomnographic data before and a few months after surgery showed significant improvements in all respiratory parameters with a greater improvement in the respiratory disturbance index in non-REM compared with REM sleep and significant improvements in the arousal index (29).

The overnight cardiorespiratory data gave an indication of the severity of SRBD in the population under study, although we acknowledge that these data were taken from one night only, so the influence of night-to-night variability could not be taken into account. We also acknowledge that, in the absence of accurate sleep staging in the recording, the influence of repeated micro-arousals on the severity of nighttime disturbance could not be taken into account. The majority of the children rated in the mild SRBD category, with very few having an $\mathrm{AHI}>5$, suggesting that the group might not be representative of the true clinical population. We did not specifically exclude those with severe SRBD, but the public hospital points referral system may have meant that those with severe symptoms received immediate treatment or sought private treatment without linking into the hospital waiting list. The range of waiting time for surgery over the study period was between 3 and 6 mo. The finding that the AHI $\geq 1.5$ was mildly associated with hyperactivity deserves further investigation in children with more severe SRBD, although two studies have found more adverse associations of externalizing behaviors in children with mild OSA compared with moderate-to-severe OSA $(21,30)$. In one study, severity of SRBD has been linked to severity of verbal impairment but to very few other neurocognitive outcomes (21).

Our findings do not allow us to draw conclusions as to possible mechanism/s whereby SRBD could contribute to behavioral problems or attentional difficulties. Findings from many studies report that chronic or intermittent hypoxemia in SRBD, even at a mild level, can adversely impact on children's behavior, cognition, and academic performance $(6,10,25,31)$. A recent study by Urschitz et al. (32) has shown that the specific variable of nocturnal $\mathrm{SaO}_{2}$ nadir is associated with impaired academic performance. Beebe and Gozal (11) have proposed a theoretical model that suggests that intermittent hypoxemia and sleep fragmentation may act synergistically to affect daytime behavior and cognitive functioning. A reduction in REM sleep, a feature of sleep fragmentation, has been linked to impaired consolidation of learned tasks and may contribute to the severity of behavioral problems in children (33). In the absence of EEG data, we cannot comment as to whether sleep disturbance related to transient arousals may have influenced any of the aspects of children's daytime functioning in this study.

In conclusion, this study adds to the accumulation of evidence that neurobehavioral functioning improves in children 
after treatment of SRBD. It must be emphasized that we report statistically significant improvements in a group of behaviorally "normal" children as distinct from clinically significant improvements. A link between SRBD and clinical diagnoses of behavioral disorders is not established, but there is a growing body of evidence that children with ADHD have a higher prevalence of SRBD than the normal population $(2,31,34)$. The finding of improved behavior and attention after $\mathrm{A} / \mathrm{T}$ in the current group of children, in whom a diagnosis of behavioral disorder was specifically excluded, may have implications for the pediatric population diagnosed with the co-existence of SRBD and behavioral and attentional disorders. Studies aimed at children with these co-existing conditions, in whom the ceiling for improvement is far higher, should be an objective for future studies.

Acknowledgments. The authors thank Amanda Phillips and Rachel Sayers for research assistance and Andrew Gray for his statistical advice. We also thank the children and their parents/guardians who participated in this study.

\section{REFERENCES}

1. Marcus CL 2001 Sleep disordered breathing in children. Am J Respir Crit Care Med $164: 16-30$

2. Ali NJ, Pitson DJ, Stradling JR 1993 Snoring, sleep disturbance, and behaviour in 4-5 year olds. Arch Dis Child 68:360-366

3. Blunden S, Lushington K, Kennedy D, Martin J, Dawson D 2000 Behavior and neurocognitive performance in children aged 5-10 years who snore compared to controls. J Clin Exp Neuropsychol 22:554-568

4. Chervin RD, Archbold KH, Dillon JE, Panahi P, Pituch KJ, Dahl RE, Guilleminault C 2002 Inattention, hyperactivity, and symptoms of sleep-disordered breathing. Pediatrics 109:449-456

5. Kennedy JD, Blunden S, Hirte C, Parsons DW, Martin AJ, Crowe E, Williams D, Pamula Y, Lushington K 2004 Reduced neurocognition in children who snore. Pediatr Pulmonol 37:330-337

6. Ali NJ, Pitson D, Stradling JR 1996 Sleep disordered breathing: effects of adenotonsillectomy on behaviour and psychological functioning. Eur J Pediatr 155:56-62

7. Avior G, Fishman G, Leor A, Sivan Y, Kaysar N, Derowe A 2004 The effect of tonsillectomy and adenoidectomy on inattention and impulsivity as measured by the Test of Variables of Attention (TOVA) in children with obstructive sleep apnea syndrome. Otolaryngol Head Neck Surg 131:367-371

8. De Serres LM, Derkay C, Sie K, Biavati M, Jones J, Tunkel D, Manning S, Inglis AF, Haddad J Jr, Tampakopoulou D, Weinberg AD 2002 Impact of adenotonsillectomy on quality of life in children with obstructive sleep disorders. Arch Otolaryngol Head Neck Surg 128:489-496

9. Friedman BC, Hendeles-Amitai A, Kozminsky E, Leiberman A, Friger M, Tarasiuk A, Tal A 2003 Adenotonsillectomy improves neurocognitive function in children with obstructive sleep apnea syndrome. Sleep 26:999-1005

10. Gozal D 1998 Sleep-disordered breathing and school performance in children. Pediatrics 102:616-620
11. Beebe DW, Gozal D 2002 Obstructive sleep apnea and the prefrontal cortex: towards a comprehensive model linking nocturnal upper airway obstruction to daytime cognitive and behavioral deficits. J Sleep Res 11:1-16

12. Brodsky L 1989 Modern assessment of tonsils and adenoids. Pediatr Clin North Am 36:1551-1569

13. American Thoracic Society 1996 Standards and indications for cardiopulmonary sleep studies in children. American Thoracic Society. Am J Resp Crit Care Med 153:866-878

14. Marcus CL, Omlin KJ, Basinki DJ, Bailey SL, Rachel AB, Von Pechmann WS, Keens TG, Ward SL 1992 Normal polysomnographic values for children and adolescents. Am Rev Respir Dis 146:1235-1239

15. Witmans MB, Keens TG, Davidson Ward SL, Marcus CL 2003 Obstructive hypopneas in children and adolescents: normal values. Am J Resp Crit Care Med 168:1540

16. Brouilette R, Hanson D, David R, Klemka L, Szatkowski A, Fernbach S, Hunt C 1984 A diagnostic approach to suspected obstructive sleep apnea in children. J Pediatr 105:10-14

17. Reynolds CR, Kamphaus RW 1992 Behavior Assessment System for Children (BASC). American Guidance Service Inc., Circle Pines, MO

18. Conners CK 2000 Conners' Continuous Performance Test II. Technical Guide and Software Manual. Multi-Health Systems Inc., Toronto, ON, Canada

19. Keith RW 1994 Auditory Continuous Performance Test (ACPT). The Psychological Corporation, San Antonio, TX

20. Cole TJ, Bellizzi MC, Flegal KM, Dietz WH 2000 Establishing a standard definition for child overweight and obesity worldwide: international survey. BMJ 320:12401243

21. Lewin DS, Rosen RC, England SJ, Dahl RE 2002 Preliminary evidence of behavioral and cognitive sequelae of obstructive sleep apnea in children. Sleep Med 5-13

22. Owens J, Opipari L, Nobile C, Spirito A 1998 Sleep and daytime behavior in children with obstructive sleep apnea and behavioral sleep disorders. Pediatrics 102:1178-1184

23. Gozal D, Pope DW Jr, 2001 Snoring during early childhood and academic performance at ages thirteen to fourteen years Pediatrics 107:1394-1399

24. Potsic WP, Pasquariello PS, Baranak CC, Marsh RR, Miller LM 1986 Relief of upper airway obstruction by adenotonsillectomy. Otolaryngol Head Neck Surg 94:476-480

25. Stradling JR, Thomas G, Warley AR, Williams P, Freeland A 1990 Effect of adenotonsillectomy on nocturnal hypoxaemia, sleep disturbance, and symptoms in snoring children. Lancet 335:249-253

26. Suen JS, Arnold JE, Brooks LJ 1995 Adenotonsillectomy for treatment of obstructive sleep apnea in children. Arch Otolaryngol Head Neck Surg 121:525-530

27. Goldstein NA, Post JC, Rosenfeld RM, Campbell TF 2000 Impact of tonsillectomy and adenoidectomy on child behavior. Arch Otolaryngol Head Neck Surg 126:494498

28. Harvey JM, O'Callaghan MJ, Wales PD, Harris MA, Masters IB 1999 Six-month follow-up of children with obstructive sleep apnoea. J Paediatr Child Health 35:136-139

29. Tal A, Bar A, Leiberman A, Tarasiuk A 2003 Sleep characteristics following adenotonsillectomy in children with obstructive sleep apnea syndrome. Chest 124:948-953

30. Harsh JR, Mixon MM, Avis KT, LeBourgeois MK 2001 Sleep, daytime sleepiness, and clinical subtypes of ADHD. Sleep 24:A15

31. O'Brien LM, Holbrook CR, Mervis CB, Klaus CJ, Bruner JL, Raffield TJ, Rutherford J, Mehl RC, Wang M, Tuell A, Hume BC, Gozal D 2003 Sleep and neurobehavioral characteristics of 5- to 7-year-old children with parentally reported symptoms of attention-deficit/hyperactivity disorder. Pediatrics 111:554-563

32. Urschitz MS, Wolff J, Sokollik C, Eggebrecht E, Urschitz-Duprat PM, Schlaud M, Poets CF 2005 Nocturnal arterial oxygen saturation and academic performance in a community sample of children. Pediatrics 115:e204-e209

33. O'Brien LM, Ivanenko A, Crabtree VM, Holbrook CR, Bruner JL, Klaus CJ, Gozal D 2003 Sleep disturbances in children with attention deficit hyperactivity disorder. Pediatr Res 54:237-243

34. Chervin RD, Dillon JE, Bassetti C, Ganoczy DA, Pituch KJ 1997 Symptoms of sleep disorders, inattention, and hyperactivity in children. Sleep 20:1185-1192 